\title{
Microfungi isolated from wood of Scots pine in Finland
}

\author{
SIRKKA SAVONMÄKI, ARVI SALONEN † and ANNA-LIISA RUOKOLA
}

\begin{abstract}
SAVONMÄKI, S., SALONEN, A. \& RUOKOLA, A-L. 1992: Microfungi isolated from wood of Scots pine in Finland. - Karstenia 32:65-70.

Non-basidiomycetous fungi were isolated from the wood of Scots pine (Pinus sylvestris) from 30 localities in Finland. They include blue-stain fungi, soil fungi and coprophilous and nematophagous fungi. The commonest fungus species were wind-borne blue-staining fungi. Fungi occurring with a frequency of more than $1 \%$ were Aureobasidium pullulans (de Bary) Annaud, Cladosporium cladosporioides (Fres.) de Vries, Geomyces pannorum (Link) Sigler \& Carm., Leptodontidium beauverioides (de Hoog) de Hoog, Oidiodendron cerealis (Thüm.) Barron, Ophiostoma piceae (Münch) H. \& P. Syd., O piliferum (Fr.) H. \& P. Syd., Phialophora fastigiata (Lagerb. \& Melin) Conant, Rhinocladiella atrovirens Nannf., Trichoderma polysporum (Link) Rifai, $T$. viride Pers. and Ulocladium consortiale (Thüm.) Simmons. As many as 35 species are apparently new to Finland, three of these belonging to the Zygomycotina, six to the Ascomycotina and 26 to the Deuteromycotina.

Key words: blue-stain fungi, microfungi, Pinus, wood
\end{abstract}

Sirkka Savonmäki \& Anna-Liisa Ruokola. Department of Plant Biology, Plant Pathology, University of Helsinki, SF-00710 Helsinki, Finland

\section{Introduction}

Most studies on the microfungi living in the wood of Scots pine (Pinus sylvestris) have dealt with harmful fungi, such as the extensively investigated blue-stain fungi, (e.g. Lagerberg et al. 1927, Rennerfelt 1950, Mathiesen-Käärik 1953, 1960, Pechmann et al. 1964, Dowding 1969, 1970, Käärik 1980, Lilja 1984). The succession and interactions of basidiomycetous fungi in wood have attracted the attention of many researchers (Butcher 1968, Käärik 1975, Rayner \& Boddy 1988). The hyphomycetous species in Canada have been studied by Sutton (1973), whose material was collected from many plant species, not especially from conifers. However, the microfungi in the wood of Scots pine are still poorly known.

A project to study microfungi in wood was started in Finland at the end of the 1960s. The original purpose was to identify blue-staining fungi in the wood of Scots pine, but later attention was paid to all the microfungi (Zygomycotina, Ascomycotina and
Deuteromycotina). The fungi inhabiting Scots pine in northern Finland were of special interest. The collection and identification of the material were completed by 1972 , but its publication has had to wait 20 years and the senior author, A. Salonen is no longer with us. This paper contains only a part of the results, because during these years some of the notes on the material have been lost and some were not found until the manuscript had already been written. The original drawings and descriptions of the fungi in Finnish have been published elsewhere (Salonen \& Ruokola 1991).

\section{Material and methods}

The material consisted of samples collected from 30 sites throughout Finland in 1968-1971. The samples were taken aseptically with an increment borer from timber, logging wastes, stumps and the wastes of sawmills. They were taken both from fresh trees 


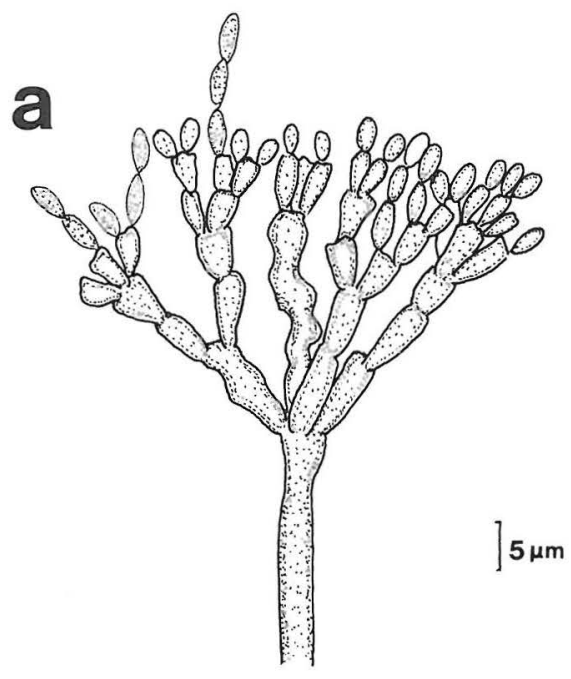

b
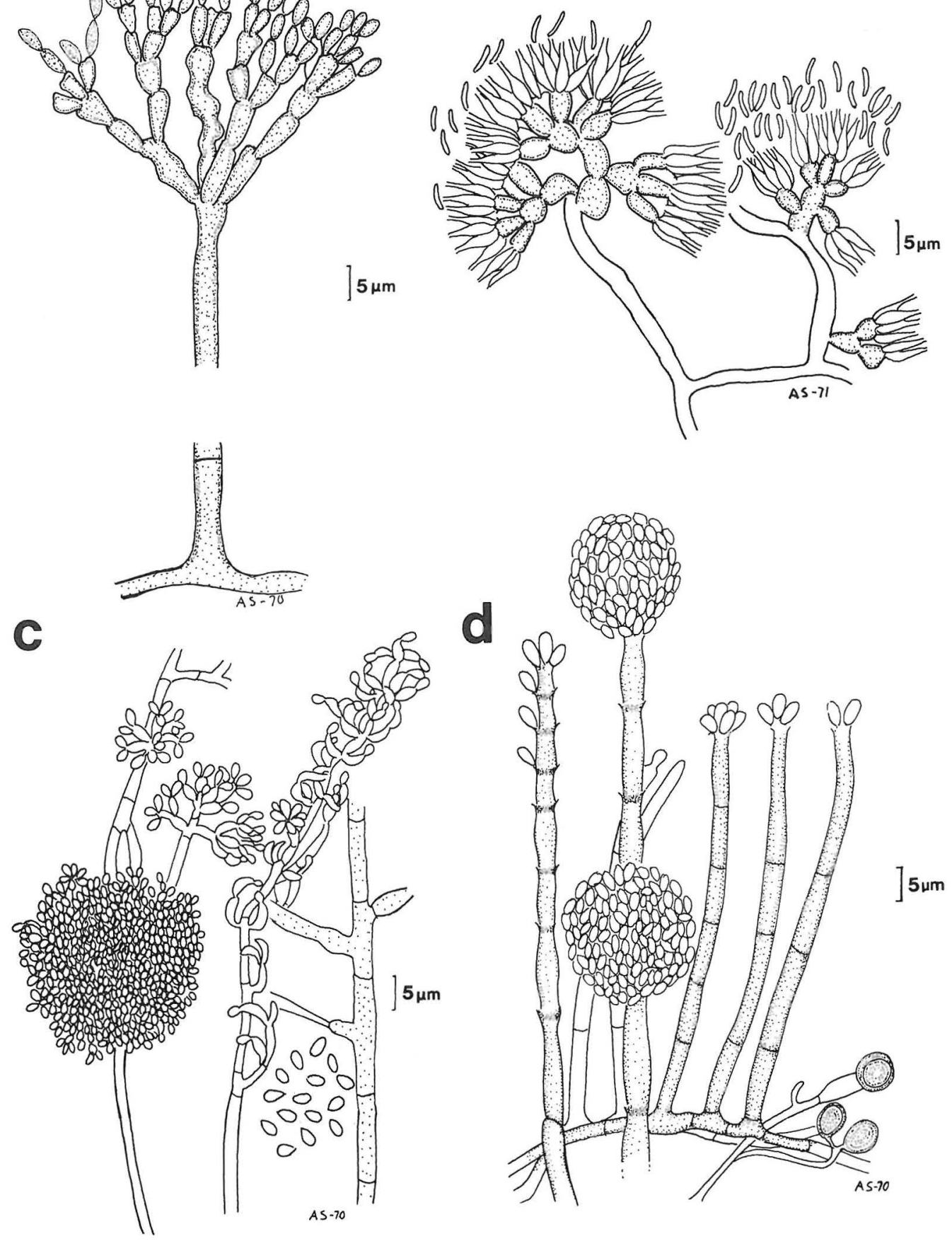

Fig. 1. Microfungi isolated from the wood of Scots pine in Finland. a. Cladosporium variabile. South Häme: Jämsä, Alhojärvi. Isol. 483.69. b. Phialophora repens. Inari Lapland: Inari, Kaamanen. Isol. 1132.70. c. Leptodontidium beauverioides. Inari Lapland: Inari, Ivalo. Isol. 643.69. d. Chloridium virescens var. chlamydosporum. Sompio Lapland: Sodankylä, Vuotso. Isol. 958.70. Drawn by Arvi Salonen. 


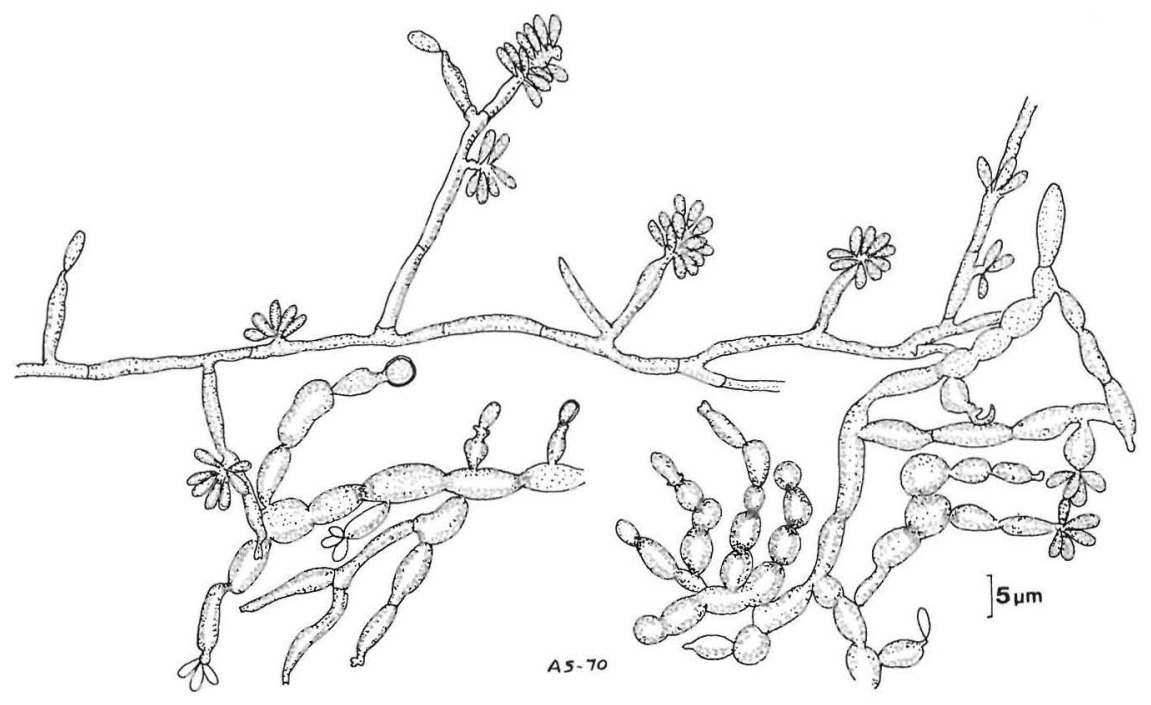

Fig. 2. Rhinocladiella spinifera. Inari Lapland: Inari, Kaamanen. Isol. 888.70. Drawn by Arvi Salonen.

felled during the current year and from timber which had been in the forest for several years. The condition of the trees furnishing the samples e.g. the moisture and degree of decomposition of the wood, is unknown.

The bore chips taken from the sample wood were transferred to corn meal agar (CMA). Pure cultures were grown on potato dextrose agar (PDA) and on CMA to which had been added $2 \%$ glucose. Malt extract agar (MEA) and Czapek agar were also used. The fungi were also cultured on autoclaved wood chips in test tubes. The cultures were kept in normal room temperature, $21-22^{\circ} \mathrm{C}$. Some fungi were identified directly from the wood samples.

Fungi were isolated from 310 samples, 178 samples $(57 \%)$ of which originated from Lapland, 142 $(46 \%)$ having been taken in Inari (c. $68^{\circ} 40^{\prime} \mathrm{N}, 27^{\circ} 33^{\prime}$ E), chiefly in the vicinity of Helsinki University Experimental Farm at Muddusjärvi. All the notes on this work are deposited in the Department of Plant Pathology, University of Helsinki, but no specimens or slide collections have been preserved.

The identifications were primarily made by Arvi Salonen. Most of the Ascomycotina were identified by Anna-Liisa Ruokola, who also conducted much of the culturing work. Sirkka Savonmäki compiled the material and wrote the present manuscript in cooperation with Ruokola. As far as possible, the nomenclature of the fungi in this paper follows Ellis (1971), Gams \& Holubová-Jechová (1976), Hoog (1977), Oorschot (1980), Sutton (1980), Cannon et al. (1985), Wingfield (1985) and Ellis \& Ellis (1988).

\section{Results}

The numbers of fungi isolated from the wood of Scots pine are given in Table 1. The isolates totalled 804, 33 of them being Zygomycotina (4.1\%), 115 Ascomycotina $(14.3 \%)$ and 656 Deuteromycotina $(81.6 \%)$. The fungi comprise 145 taxa. The Zygomycotina are represented by 11 taxa (7.8\%), the Ascomycotina by 25 (17.7\%) and the Deuteromycotina by 105 (74.5\%). The fungi which could be identified to species level numbered 104, most of them being Deuteromycotina (Figs. $1 \& 2$ ). The number of species apparently new to Finland is 35 (3 Zygomycotina, 6 Ascomycotina and 26 Deuteromycotina).

\section{Discussion}

The fungi living in Scots pine mainly depend on the condition of the wood, for example the degree of decomposition. The material was collected from both fresh and older timber left in forests and from the wastes of sawmills. Some of the species were primary fungi attacking fresh timber, but most of them were secondary saprophytic fungi, common in debris and also in dung. Besides these, there were some nematophagous fungi. Most of the fungus species occurring with a frequency of at least $1 \%$ in the wood of Scots pine (Table 1) were air-borne blue-staining fungi (Zimmermann \& Butin 1973).

The isolates belonging to the Zygomycotina and Ascomycotina include especially many species normally occurring in dung (e.g. Sporormiella) (Ahmed 
Table 1. Fungi isolated from wood samples of Scots pine (Pinus sylvestris). The figures refer to the number of samples in which each species was recorded.

\begin{tabular}{cc}
\hline Fungus & No of samples recorded \\
& I II III
\end{tabular}

\section{ZYGOMYCOTINA}

Absidia glauca Hagem

* Circinella simplex van Tieghem

Circinella sp.

* Mortierella polycephala Coemans

Mortierella spp.

Mucor mucedo (L.) Fres.

Mucor racemosus Fres.

Mucor spp.

Rhizopus stolonifer (Ehrenb.) Vuill.

* Syncephalastrum racemosum Cohn ex Schröt0 1

Thamnidium elegans Link

\begin{tabular}{lllll} 
& 2 & 0 & 1 & 3 \\
& 0 & 3 & & 3 \\
& 1 & 0 & & 1 \\
1 & 0 & & 1 \\
& 6 & 3 & & 9 \\
& 2 & 1 & & 3 \\
& 0 & 4 & & 4 \\
& 0 & 6 & & 6 \\
& 0 & 1 & & 1 \\
& \\
ex Schröt0 & 1 & & 1 \\
& 0 & 1 & & 1 \\
\hline$\Sigma$ & 12 & 20 & 1 & 33
\end{tabular}

\section{ASCOMYCOTINA}

Cephaloascus fragrans Hanawa

Ceratocystis coerulescens (Münch) Bakshi

Ceratocystis / Ophiostoma spp.

Chaetomium elatum Schm. \& Kunze

Chaetomium globosum Kunze

Chaetomium spirale Zopf

Chaetomium sp.

* Coniochaeta ligniaria (Grev.) Cooke

Coniochaeta spp.

Gelasinospora tetrasperma Dowding

Gelasinospora sp.

Gymnoascus reessii Baranetzky

Melanospora sp.

* Myxotrichum berkeleyi Apinis

* Ophiostoma minus (Hedg.) H. \& P. Syd.

(= Ceratocystis minor (Hedg.) Hunt)

Ophiostoma piceae (Münch) H. \& P. Syd.

(= Ceratocystis piceae (Münch) Bakshi)

Ophiostoma piliferum (Fr.) H. \& P. Syd.

(= Ceratocystis pilifera (Fr.) C. Moreau)

* Schizothecium vesticola

(Berk. \& Broome) Lundq.

(= Pleurage minuta (Fuckel) Kunze)

Sordaria fimicola (Rob. ex Desm.)

Ces. \&de Not.

Sordaria macrospora Auersw.

* Sphaerodes fimicola (Hansen) P. Cannon

\& Hawsksw.

Sporormiella intermedia (Auersw.)

Ahmed \&Cain

(= Sporormia intermedia Auersw.)

* Sporormiella octomera (Auersw.)

Ahmed \& Cain

(= Sporormia octomera Auersw.)

Sporormiella sp.

(= Sporormia sp.)

Thelebolus crustaceus (Fuckel) Kimb.

(= Pezizula crustacea (Fuckel) Karst.)

$\overline{\sum \quad 42 \quad 67 \quad 6115}$

Table 1. Contnd.

\begin{tabular}{cc}
\hline Fungus & No of samples recorded \\
I II III $\Sigma$
\end{tabular}

DEUTEROMYCOTINA

Acremonium butyri (van Beyma) W. Gams $\quad 0066$

* Acremonium roseum (Oud.) W. Gams $\quad 0011$

Acremonium spp.

$\begin{array}{lll}6 & 21 & 27\end{array}$

* Acrodontium crateriforme (van Beyma)

de Hoog

(= Chloridium crateriforme van Beyma)

Alternaria alternata (Fr.) Keissler

Alternaria sp.

* Amblyosporium botrytis Fres.

$\begin{array}{llll} & & & 2\end{array}$

Arthim phaeospermum (Corda) M.B. Ellis

(= Papularia sphaerosperma (Pers.) Höhnel)

Arthrobotrys superba Corda

Aspergillus fumigatus Fres.

Aspergillus niger van Tieghem

Aspergillus spp.

Aureobasidium pullulans (de Bary) Arnaud

* Beauveria nivea (Rostrup) von Arx

$\begin{array}{lll}0 & 1 & 1\end{array}$

(= Pachybasium niveum Rostrup)

(= Tolypocladium inflatum W. Gams)

Bloxamia sp.

* Botryotrichum piluliferum Sacc. \& March.

Botrytis cinerea Pers.

Botrytis sp.

Chalara sp.

$\begin{array}{lll}1 & 0 & 1\end{array}$

$\begin{array}{lll}4 & 0 & 4\end{array}$

$\begin{array}{lll}0 & 1 & 1\end{array}$

$\begin{array}{lll}6 & 9 & 15\end{array}$

$\begin{array}{lll}9 & 22 & 31\end{array}$

Cheiromycella microscopica (Karst.) Hughes $1 \quad 0 \quad 1$

* Chloridium virescens (Pers.) W. Gams \& Hol.- Jech.

var. chlamydosporum (van Beyma)

W. Gams \& Hol.-Jech.

(=C. chlamydosporis (van Beyma) Hughes)

* Chloridium virescens (Pers.) W. Gams \&

Hol.-Jech. var. virescens

(=C. viride Link)

Chloridium (s.lat.) spp.

$\begin{array}{lll}1 & 0 & 1\end{array}$

0

$\begin{array}{ll}0 & 1\end{array}$

$0 \quad 1$

420

1

1

24

Chromelosporium fulvum (Link) McGinty et al.3 003

$\begin{array}{llll}\text { Chrysosporium (s.lat.) spp. } & 0 & 2 & 2\end{array}$

Cladosporium cladosporioides (Fres.) de Vries10 $13 \quad 23$

$\begin{array}{llrr}\text { Cladosporium herbarum (Pers.) Link } & 0 & 2 & 2\end{array}$

* Cladosporium macrocarpum Preuss $\quad 0011$

$\begin{array}{llll}\text { Cladosporium sphaerospermum Penz. } & 3 & 1 & 4\end{array}$

* Cladosporium variabile (Cooke) de Vries $\begin{array}{llll}1 & 0 & 1\end{array}$

$\begin{array}{llll}\text { Cladosporium spp. } & 9 & 13 & 22\end{array}$

$\begin{array}{llll}\text { Cordana pauciseptata Preuss } & 0 & 3 & 3\end{array}$

$\begin{array}{llll}\text { Cylindrocarpon sp. } & 2 & 3 & 5\end{array}$

$\begin{array}{llll}\text { Dactylella gephyropaga Drechsler } & 1 & 0 & 1\end{array}$

$\begin{array}{llll}\text { Dendrodochium sp. } & 1 & 1 & 2\end{array}$

Doratomyces nanus (Ehrenb.) Morton \& Smith0 2

Doratomyces stemonitis (Pers.) Morton \&

Smith

Epicoccum purpurascens Ehrenb.

$\begin{array}{lll}0 & 2 & 2\end{array}$

(=E. nigrum Link )

Fusarium culmorum (W.G. Sm.) Sacc. $\quad 1 \quad 0 \quad 1$

Fusarium moniliforme Sheld. $\quad 2 \quad 0 \quad 2$

Fusarium oxysporum Schlecht. $\quad 1 \quad 001$

Fusarium poae (Peck) Wollenw. $\quad 0 \quad 1 \quad 1$

Fusarium sp.

Fusidium sp.
(Contnd.) 
Table 1. Contnd.

Fungus

No of samples recorded

I II III $\Sigma$

Geomyces pannorum (Link) Sigler \& Carm.

var. pannorum

$\begin{array}{llll}1 & 11 & 1 & 13\end{array}$

(= Chrysosporium pannorum (Link) Hughes)

Geotrichum candidum Link

Gliocladium viride Matr.

(= G. deliquescens Sopp)

Gliocladium roseum (Link) Bainier

Graphium spp.

* Helminthophora sphaerocephala (Berk.)

de Hoog

(= Cladobotryum leptosporum (Sacc.)

W. Gams \& Hoozemans)

Leptodontidium beauverioides

(de Hoog) de Hoog

(= Leptodontium beauverioides de Hoog)

* Leptographium antibioticum (Kendrick)

Wingfield

(=Verticicladiella antibiotica Kendrick)

Leptographium brachiatum (Kendrick)

Wingfield

(=Verticicladiella brachiata Kendrick)

Leptographium lundbergii Lagerb. \& Melin

Leptographium procerum (Kendrick) Wingfield3

(=Verticicladiella procera Kendrick)

Leptographium sp.

* Malbranchea pulchella Sacc. \& Penz.

Monodictys levis (Wiltshire) Hughes

Monodictys sp.

* Myceliophthora vellerea (Sacc. \& Speg.)

van Oorschot

(= Chrysosporium asperatum Carm.)

* Myrothecium verrucaria (Alb. \& Schw.)

Ditm.: Fr.

Nematoctonus lignicola Salonen \& Ruokola,

nom. inval.

Oedocephalum sp.

Oidiodendron cerealis (Thüm.) Barron

Oidiodendron griseum Robak

* Oidiodendron maius Barron

* Oidiodendron rhodogenum Robak

* Oidiodendron tenuissimum (Peck) Hughes

Oidiodendron spp.

Paecilomyces penicillatus (Höhnel) Samson

(= Spicaria elegans (Corda) Harz)

Paecilomyces variotii Bainier

Paecilomyces sp.

* Penicillium thomii Maire

Penicillium spp.

$\begin{array}{llll}1 & 22 & 2 & 25\end{array}$
Table 1. Contnd.

Fungus

No of samples recorded

I II III $\Sigma$

* Phaeostalagmus tenuissimus (Corda)

W. Gams

(=Verticillium tenuissimum Corda)

* Phialophora cinerescens (Wollenw.)

van Beyma

Phialophora fastigiata (Lagerb. \& Melin)

Conant

Phialophora lagerbergii (Melin \& Nannf.)

Conant

Phialophora lignicola (Nannf.) Goidanich

* Phialophora repens (Davidson) Conant

* Phialophora richardsiae (Nannf.) Conant

Phialophora verrucosa Medlar

Phialophora spp.

Phoma herbarum Westend.

Phoma spp.

$\begin{array}{llll}0 & 0 & 1 & 1\end{array}$

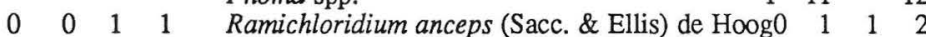

(= Rhinocladiella anceps (Sacc. \& Ellis) Hughes)

Rhinocladiella atrovirens Nannf.

$\begin{array}{llll}6 & 13 & 2 & 21\end{array}$

Rhinocladiella spinifera (Nielsen \& Conant)

de Hoog

(=R. mansonii (Castell) Schol-Schwarz)

Rhinocladiella spp.

Sclerophoma sp.

Sporothrix sp.

Stemphylium botryosum Westend.

$\begin{array}{llll}1 & 3 & 1 & 5\end{array}$

$\begin{array}{llll}5 & 27 & 1 & 33\end{array}$

$\begin{array}{lll}2 & 2 & 4\end{array}$

$12-2$

$\begin{array}{llll}1 & 4 & 1 & 5 \\ 2 & 3 & & 5\end{array}$

$\begin{array}{lll}0 & 4 & 4\end{array}$

$\begin{array}{lll}7 & 17 & 24\end{array}$

$\begin{array}{llll}1 & 4 & 1 & 6\end{array}$

$11 \quad 12$

Sterigmatobotrys macrocarpa (Corda) Hughes 0

* Thysanophora penicilloides (Roum.) Kendrick1 0

Tilachlidium sp. $\quad 0 \quad 2202$

$\begin{array}{llll}\text { Torula sp. } & 5 & 14 & 19\end{array}$

Trichocladium asperum Harz $\quad 1 \quad 2 \quad 3$

Trichocladium opacum (Corda) Hughes $\quad \begin{array}{lll}1 & 0 & 1\end{array}$

$\begin{array}{llll}\text { Trichoderma polysporum (Link) Rifai } & 5 & 7 & 12\end{array}$

$\begin{array}{llll}\text { Trichoderma viride Pers. } & 11 & 11 & 22\end{array}$

$\begin{array}{llll}\text { Trichoderma sp. } & 6 & 2 & 8\end{array}$

Truncatella angustata (Pers.) Hughes $\quad 0011$

(=T. truncata (Lév.) Stey.)

$\begin{array}{llll}\text { Ulocladium consortiale (Thüm.) Simmons } & 4 & 7 & 11\end{array}$

(= Stemphylium consortiale (Thüm.) Groves \&

Skolko)

*Wardomyces anomalus Brooks \& Hansf. $\quad 0 \quad 3 \quad 3$

$\sum \quad 197446 \quad 13656$

$==================$

$\mathrm{I}=$ South and Central Finland

II = Lapland

III = unknown collecting site

(Contnd.) * = species new to Finland
\& Cain 1972), and common in soil (Domsch et al.1980). Though mucoraceous fungi are usually isolated from soil or dung they are also associated with wood. Mortierella species have been isolated from wood at different stages of decomposition (Rayner \&
Boddy 1988), and Merrill \& French (1966) isolated Mucor, Absidia and Rhizopus species from wood buried in soil. In this material, Zygomycotina did not occur abundantly. Some fungi were typical of wood in contact with soil. Aspergillus spp., Alternaria spp., 
Trichoderma viride and Penicillium spp. are among the first invaders of wood in soil and have earlier been isolated from Ponderosa pine (Pinus ponderosa) (Merrill \& French 1966). In Butcher's (1968) study of the succession of fungi colonizing untreated stakes, the most common species were Trichoderma viride, Fusarium spp., Epicoccum purpurascens and Alternaria tenuis. The present isolates also include fungicolous species, such as Helminthophora sphaerocephala (Hoog 1978).

Nematophagous fungi were rarely isolated in our study (Table 1). Three species were identified: $A r$ throbotrys superba, Dactylella gephyrophaga and Nematoctonus lignicola. $N$. lignicola has been described from Finland (Salonen \& Ruokola 1968), but was not validly published (Thorn \& Barron 1986).

The fungi living in the wood of Scots pine have not been widely studied previously in Finland. Although no definite conclusions can be drawn about their occurrence on the basis of this material, certain species appear to be concentrated in Lapland. For example, most of the samples of Leptodontidium beauverioides were from Lapland. This species has earlier been isolated from the wood of Norway spruce (Picea abies) in Finland (Hoog 1977, 1979).

Acknowledgements. We wish to thank all the persons who have taken part in this research. We are particularly indebted to Pentti Heinänen, for technical assistance. We are grateful to Hilkka Koponen and Lalli Laine for their advice and kind support during the writing of the manuscript. The language of the manuscript was revised by Ms. Anna Damström. The Foundation for Research of Natural Resources in Finland gave financial support for the preparation of this paper.

\section{References}

Ahmed, S. I. \& Cain, R. F. 1972: Revision on the genus Sporormia and Sporormiella. - Canadian J. Bot. 50:419477.

Butcher, J. A. 1968: The ecology of fungi infecting untreated sapwood of Pinus radiata. - Canadian J. Bot. 46:15771589.

Cannon, P. F., Hawksworth, D. L. \& Sherwood-Pike, M. A. 1985: The British Ascomycotina. An annotated checklist. 302 pp. Commonwealth Mycological Institute, Kew.

Domsch, K. H., Gams, W. \& Anderson, T.-H. 1980: Compendium of soil fungi. -859 pp. Academic Press, London.

Dowding, P. 1969: The dispersal and survival of spores of fungi causing bluestain in pine. - Trans. British Mycol. Soc. 52: 125-137.

Dowding, P. 1970: Colonization of freshly bared pine sapwood surfaces by staining fungi. - Trans. British Mycol. Soc. 55: $399-412$.

Ellis, M. B. 1971: Dematiaceous Hyphomycetes. - 608 pp. Commonwealth Mycological Institute, Kew.
Ellis, M. B. \& Ellis, J. P. 1988: Microfungi on miscellaneous substrates. - 244 pp. Croom Helm, London.

Gams, W. \& Holubová-Jechová, V. 1976: Chloridium and some other dematiaceous Hyphomycetes growing on decaying wood. - Stud. Mycol. 15:1-99.

Hoog, G. S. de 1977: Rhinocladiella and allied genera. - Stud. Mycol. 15:1-140.

Hoog, G. S. de 1978: Notes on some fungicolous hyphomycetes and their relatives. - Persoonia 10:33-81.

Hoog, G. S. de 1979. Nomenclatural notes on some black yeastlike Hyphomycetes. - Taxon 28:347-348.

Käärik, A. 1975: Succession of microorganisms during wood decay. In: Liese, W. (ed.), Biological transformation of wood by microorganism: 39-51. - Springer Verlag, Berlin.

Käärik, A. 1980: Fungi causing sap stain in wood. - Sveriges Lantbruksuniv. Inst. virkeslära, Rapport R 114: 1-112.

Lagerberg, T., Lundberg, G. \& Melin, E. 1927: Biological and practical research into blueing in pine and spruce. - Svenska Skogsvårdsför.Tidskr. 1927:145-272.

Lilja, A. 1984: Ilmalevintäisen sinistymisen aiheuttajista ja eräiden fungisidien tehosta niiden torjunnassa. - Folia Forest. 592:1-12.

Mathiesen-Käärik, A. 1953: Eine Übersicht über die gewöhnlichen mit Borkenkäfern assoziierten Bläuepilze in Schweden und einige für Schweden neue Bläuepilze. - Medd. Stat. Skogsforskn. inst. 43:1-74.

Mathiesen-Käärik, A. 1960: Studies on the ecology, taxonomy and physiology of Swedish insect-associated blue-stain fungi, especially the genus Ceratocystis. - Oikos 11:1-25.

Merrill, W. \& French, D. W. 1966: Colonization of wood by soil fungi. - Phytopathology 56:301-303.

Oorschot, C. A. N. van 1980: A revision of Chrysosporium and allied genera. - Stud. Mycol. 20:1-89.

Pechmann, H. von, Graessle, E. \& Wutz, A. 1964: Untersuchungen über Bläuepilze an Kiefernholz. - Forstwiss. Centralbl. 83:290-314.

Rayner, A. D. M. \& Boddy, L. 1988: Fungal decomposition of wood. Its biology and ecology. - 587 pp. John Wiley \& Sons, Chichester.

Rennerfelt, E. 1950: Über den Zusammenhang zwischen dem Verblauen des Holzes und den Insekten. - Oikos 2:120 137.

Salonen, A. \& Ruokola, A-L. 1968: On nematode-destroying fungi in Finland. II. - J. Sci. Agric. Soc. Finland 40:142145.

Salonen, A. \& Ruokola, A.-L. 1991: Piirroskokoelma mäntypuutavaran mikrosienistä Suomessa. - Helsingin yliopiston kasvipatologian lait. monist. 62:1-76.

Sutton, B. C. 1973: Hyphomycetes from Manitoba and Saskatchewan, Canada. - Mycol. Pap. 132:1-143.

Sutton, B. C. 1980: The Coelomycetes. Fungi imperfecti with pycnidia, acervuli and stromata. - $696 \mathrm{pp}$. Commonwealth Mycological Institute, Kew.

Thorn, R. G. \& Barron, J. L. 1986: Nematoctonus and the tribe Resupinateae in Ontario, Canada. - Mycotaxon 25:321453.

Wingfield, M. J. 1985: Reclassification of Verticicladiella based on conidial development. - Trans. British Mycol. Soc. 85:81-93.

Zimmermann, G. \& Butin, H. 1973: Untersuchungen über Hitze- und Trockenresistenz holzbewohnender Pilze. Flora 162:393-419.

Received on 18 January 1991 